\title{
Freeze-Dried Platelet-Rich Plasma Induces Osteoblast Proliferation via Platelet-Derived Growth Factor Receptor-Mediated Signal Transduction
}

\author{
Hideyuki Kinoshita ${ }^{1,2}$, Sumihisa Orita ${ }^{1}$, Kazuhide Inage ${ }^{1}$, Kazuki Fujimoto ${ }^{3}$, Yasuhiro Shiga ${ }^{1}$, Koki Abe ${ }^{1}$, \\ Masahiro Inoue ${ }^{1}$, Masaki Norimoto ${ }^{1}$, Tomotaka Umimura ${ }^{1}$, Takeshi Ishii ${ }^{2}$, Tsukasa Yonemoto ${ }^{2}$, \\ Hiroto Kamoda ${ }^{2}$, Toshinori Tsukanishi ${ }^{2}$, Masahiko Suzuki ${ }^{1}$, Naoya Hirosawa ${ }^{1}$, \\ Tsutomu Akazawa ${ }^{4}$, Seiji Ohtori ${ }^{1}$ \\ ${ }^{1}$ Department of Orthopaedic Surgery, Graduate School of Medicine, Chiba University, Chiba, Japan \\ ${ }^{2}$ Department of Orthopedic Surgery, Chiba Cancer Center, Chiba, Japan \\ ${ }^{3}$ Department of Orthopaedic Surgery, Saiseikai Narashino Hospital, Narashino, Japan \\ ${ }^{4}$ Department of Orthopaedic Surgery, School of Medicine, St. Marianna University, Kawasaki, Japan
}

\section{Study Design: Controlled laboratory study.}

Purpose: This study aimed to evaluate the in vitro pharmacological activity of growth factors (GFs) in freeze-dried platelet-rich plasma (FD-PRP) after storage for 4 weeks.

Overview of Literature: Freshly prepared PRP is a rich source of many GFs. We reported that FD-PRP stored for 8 weeks accelerated bone union in a rat posterolateral fusion model equally well as fresh-PRP. However, the pharmacological activity of FD-PRP after longterm storage has not been shown in vitro.

Methods: Immediately after preparation, as well as 4 weeks after freeze-dried storage, the platelet count was measured. Human osteoblasts were treated with fresh-PRP and FD-PRP, respectively. Western blotting was used to assess the phosphorylation of the platelet-derived growth factor (PDGF) receptor (PDGFR) and its downstream target, extracellular signal-regulated kinase (ERK). The proliferation rates of osteoblasts were investigated by immunocytochemistry and MTT cell viability assays. Furthermore, we used western blotting to evaluate the effect of PDGFR knockdown on the phosphorylation of ERK stimulated with fresh-PRP and FD-PRP.

Results: Platelet counts in both the fresh-PRP and FD-PRP samples were approximately 10-fold higher than in peripheral blood samples. The phosphorylation and activation of the PDGFR and ERK were evenly induced by fresh-PRP and FD-PRP stimulation. Both freshPRP and FD-PRP significantly induced osteoblast proliferation in MTT cell viability assays. Furthermore, osteoblast PDGFR knockdown attenuated the downstream ERK activation by fresh PRP and FD-PRP.

Conclusions: We demonstrated the pharmacological activity of PDGF in FD-PRP in vitro after 4 weeks of storage.

Keywords: Freeze-dried platelet-rich plasma; Osteoblasts; Proliferation; Platelet-derived growth factor

Received Feb 12, 2019; Revised Mar 26, 2019; Accepted Apr 13, 2019

Corresponding author: Hideyuki Kinoshita

Department of Orthopaedic Surgery, Graduate School of Medicine, Chiba University, 1-8-1 Inohana, Chuo-ku, Chiba 260-8670, Japan Tel: +81-43-226-2117, Fax: +81-43-226-2116, E-mail: kinoshi1783@yahoo.co.jp 


\section{Introduction}

Platelet-rich plasma (PRP) is an autologous blood product consisting of a high number of platelets, as well as various growth factors (GFs), in a small volume of plasma. Recently, PRP has gained attention in musculoskeletal regenerative therapies [1-3] as it clinically enhances neoangiogenesis, tissue repair, and regeneration [4]. Thus, PRP is emerging as a novel treatment for musculoskeletal pathologies, such as ligament injury, muscle tears, tendinopathies, and osteoarthritis [5,6]. For instance, intra-articular injections of autologous PRP are effective in patients with moderate osteoarthritis [7]. In previous studies, we showed that PRP accelerated bone union and induced a reduction of the bone union period in both rats and humans with spinal posterolateral fusion (PLF) [8-10]. It appears that platelet-derived growth factor (PDGF) plays an essential role in such effects as it has the ability to promote bone fusion [11]. PDGF is a member of a large family of GFs secreted by human vascular endothelial cells and fibroblasts to induce phosphorylation and activation of the PDGF receptor (PDGFR), which thereby activates downstream pathways that regulate cell growth and division.

During the clinical PRP application, one of the major issues that do not allow for long-term PRP storage is the relatively short half-life of GFs in PRP. Thus, patients are required to donate a large amount of blood just before surgery for fresh PRP preparation. To overcome this limitation, we focused on freeze-dried PRP (FD-PRP) and confirmed that FD-PRP maintained baseline levels of GFs for the entire 8-week duration [12]. Furthermore, Shiga et al. [13] showed that FD-PRP could achieve accelerated bone union in a rat PLF model even after 8-week storage. However, there is no report showing the pharmacological activity of GFs, such as PDGF, in FD-PRP in storage for a long-term period. In the current study, we investigated the pharmacological activity of FD-PRP in vitro after 4 weeks of storage.

\section{Materials and Methods}

\section{Fresh-platelet-rich plasma preparation}

Rather than using autograft blood, we used rodent allograft blood to prepare the PRP. After the rodents were deeply anesthetized, approximately $15 \mathrm{~mL}$ of fresh blood was obtained via transcardiac puncture using a syringe containing 2-mL acid-citrate-dextrose solution A (Terumo, Tokyo, Japan) to prevent coagulation. According to a past report for preparing PRP and platelet-poor plasma (PPP) [13-15], the whole blood was centrifuged (KN70; Kubota, Tokyo, Japan) at 1,500 rpm for 10 minutes. Subsequently, the plasma fraction was separated from the red blood cells and further centrifuged at 3,000 rpm for 10 minutes to obtain pelleted platelets as previously described. Then, the pelleted platelets were collected and separated from the supernatant PPP. The PRP was generated by mixing the pelleted platelets with $1 \mathrm{~mL} P P P$.

\section{Freeze-dried platelet-rich plasma preparation}

Each of the PRP aliquots was weighed prior to freezedrying. The test tubes were rotated in an ethanol bath at $-60^{\circ} \mathrm{C}$ for membrane freezing (preliminary freezing). Then, the test tubes were immediately frozen at $-30^{\circ} \mathrm{C}$ for 4 hours. The tubes were then attached to a vacuum freeze dryer to complete the process and stored for 4 weeks at $4^{\circ} \mathrm{C}$. Prior to assessment, the FD-PRP samples were resuspended in distilled water. To avoid any changes in component concentrations, the weight after resuspension was matched to the weight before freeze-drying. Because PRP requires activation before application, $\mathrm{CaCl}_{2}(1 \mathrm{mEq} /$ $\mathrm{mL}$; Otsuka Pharmaceutical, Tokyo, Japan) and thrombin (Mochida Pharmaceutical, Tokyo, Japan) solutions were added to both fresh-PRP and FD-PRP samples. Each added solution was one-tenth of the volume of both freshPRP and FD-PRP (Fig. 1). We used a hematology analyzer to determine the platelet counts in whole blood, freshPRP, and FD-PRP.

\section{Antibodies and reagents}

Osteoblast Growth Medium (C-27001) and Osteoblast Growth Medium Supplement Mix (C-39615) were obtained from PromoCell (Tokyo, Japan); and RIPA lysis buffer from Santa Cruz Biotechnology Inc. (Santa Cruz, CA, USA). The following antibodies were obtained from Cell Signaling Technology (Danvers, MA, USA): anti-p42/44 mitogen-activated protein kinase (MAPK) (Erk1/2) (\#9102), anti-PDGFR $\beta$ (28E1) (\#3169), antiphospho-p44/42 MAPK (Erk1/2) (Thr202/Tyr204) (\#9101), anti-phospho-PDGFR $\beta$ (Tyr 1009) (42F9) (\#3124), and anti- $\beta$-actin (\#4967). The antibody antiDAPI was obtained from Life Technologies (Carlsbad, 
CA, USA); anti-Alexa Fluor 488 and anti-Alexa 594 from Invitrogen (Carlsbad, CA, USA); and horseradish peroxidase-conjugated secondary antibodies and polyvinylidene difluoride (PVDF) western blotting membrane were from GE Healthcare (Tokyo, Japan).

\section{Platelet counts}

Platelets were counted using a K-4500 counter (Sysmex, Tokyo, Japan). To avoid deviations due to platelet aggregation, platelet counting was performed using frozen and lyophilized samples after thawing at room temperature and after 3-4 hours of resuspension in distilled water.

\section{Cell culture}

Primary human osteoblasts (PromoCell) were grown in Osteoblast Growth Medium supplemented with Supplement Mix and $100 \mu \mathrm{g} / \mathrm{mL}$ penicillin/streptomycin in a humidified $5 \% \mathrm{CO}_{2}$ atmosphere at $37^{\circ} \mathrm{C}$.

\section{RNA interference}

For RNA interference, cells at $80 \%$ confluency were transfected with the following small interfering RNAs (siRNAs; Life Technologies) using Lipofectamine RNAiMAX reagent (Life Technologies) according to the manufacturer's instructions. Silencer siRNA targeting PDGFR $\beta$ (ID no. s10242 and s10241) and silencer select negative control siRNA for PDGFR were transfected. After knockdown with each siRNA for 48 hours, siRNA-transfected cells were stimulated with fresh-PRP or FD-PRP (both with final platelet concentrations of $3 \times 10^{4} / \mu \mathrm{L}$ or $6 \times 10^{4} / \mu \mathrm{L}$ for 10 minutes) and evaluated by western blotting.

\section{Immunoblotting}

All wash buffers and extraction buffers included protease inhibitor cocktail (Roche, Tokyo, Japan), PhosSTOP phosphatase inhibitor (Roche), $\mathrm{NaF}(1 \mathrm{M})$, and Na3VO4 $(50 \mu \mathrm{M})$. Cell extracts were resolved with SDS-PAGE and electroblotted onto PVDF membranes. After blocking with 5\% skim milk in TBS-T (50 mM Tris $\mathrm{HCl}$, pH 8.0, $150 \mathrm{mM} \mathrm{NaCl}$, and $0.05 \%$ Tween-20), the membranes were probed with antibodies. The antibody-antigen complexes were detected using an enhanced chemiluminescence system (GE Healthcare).

\section{Immunocytochemical staining}

Osteoblast cells were stimulated with fresh-PRP or FDPRP (final platelet concentration of $3 \times 10^{4} / \mu \mathrm{L}$ ) or control for 48 hours. Osteoblast cells were fixed with $4 \%$ (weight/ volume $[\mathrm{w} / \mathrm{v}]$ ) formaldehyde (Wako) in phosphatebuffered saline (PBS) at room temperature for 10 minutes. Next, the samples were incubated with $0.1 \%(w / v)$ Triton X-100 in PBS for 10 minutes and subsequently with $0.5 \%(\mathrm{w} / \mathrm{v})$ skim milk in PBS at room temperature for 30 minutes. The samples were then incubated with primary antibodies overnight at $4^{\circ} \mathrm{C}$. Then, the samples were incubated with secondary antibodies at room temperature for 1 hour. The samples were counterstained with DAPI to visualize cell nuclei, and the images were processed using a fluorescence microscope.

\section{Cell viability assay}

Cultured osteoblast cells at a concentration of $1 \times 10^{6}$ cells/ $\mathrm{mL}$ in a 96-well plate were stimulated with fresh-PRP or FD-PRP (final platelet concentration of $3 \times 10^{4} / \mu \mathrm{L}$ ) or control for 48 hours. Then, 3-(4,5-dimethylthiazol-2-yl)-2,5diphenyltetrazolium bromide (MTT) solution (Cayman Chemical, Ann Arbor, MI, USA) was added at a volume equivalent to $10 \%$ of the culture volume, under sterile conditions and incubated for 4 hours in a $\mathrm{CO}_{2}$ incubator at $37^{\circ} \mathrm{C}$. After incubation, a crystal dissolving solution was added to cultures and incubated for 4-18 hours in a $\mathrm{CO}_{2}$ incubator at $37^{\circ} \mathrm{C}$. Then, the absorbance of each sample at $570 \mathrm{~nm}$ was measured using a microplate reader. Cell viability (\%) was calculated based on the following equation:

(Experimental group absorbance value/control group absorbance value) $\times 100$.

\section{Statistical analysis}

Experimental data are expressed as mean \pm standard deviation. Significant differences between mean values were calculated using Student $t$-test, with $p<0.05$ as the criterion for statistical significance.

\section{Results}

\section{Platelet counts in fresh-PRP and FD-PRP}

Before use, fresh-PRP and FD-PRP stored for 4 weeks 
were activated with $\mathrm{CaCl}_{2}$ and thrombin to stimulate human osteoblasts (Fig. 1). The mean platelet counts were $(35.9 \pm 8.3) \times 10^{4} / \mu \mathrm{L}$ in the whole blood sample, $(313 \pm 46.7) \times 10^{4} / \mu \mathrm{L}$ in the fresh-PRP, and $(335.5 \pm 4.1) \times 10^{4} /$ $\mu \mathrm{L}$ in the FD-PRP. The platelet counts in the fresh-PRP and FD-PRP samples were approximately 10 -fold higher than in peripheral blood samples, and the samples were evenly concentrated (Fig. 2).

\section{Fresh-PRP and FD-PRP activate PDGFR and its down- stream target ERK, inducing the proliferation of osteo- blasts}

Results of western blotting showed the phosphorylation and activation of the downstream protein extracellular signal-regulated kinase (ERK), as well as PDGFR in both fresh-PRP (Fig. 3A) and FD-PRP (Fig. 3B) samples. A sig-

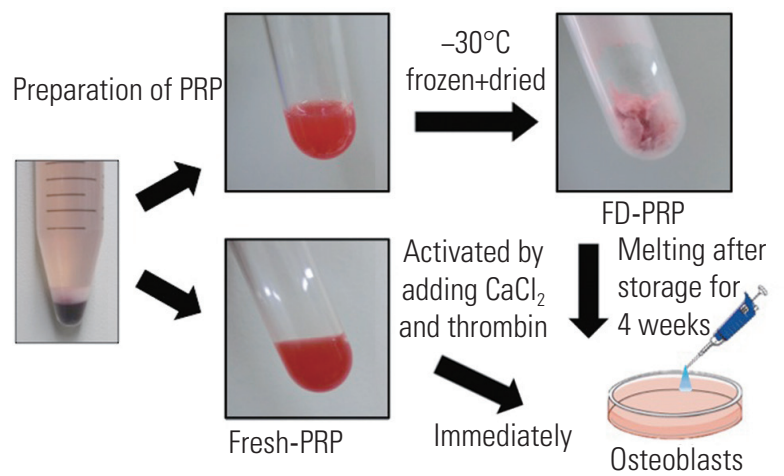

Fig. 1. Preparation of fresh-PRP and FD-PRP. FD-PRP was stored for 4 weeks and both fresh-PRP and FD-PRP were activated with $\mathrm{CaCl}_{2}$ and thrombin before use. PRP, platelet-rich plasma; FD-PRP, freeze-dried platelet-rich plasma. nificant difference in the phosphorylation of PDGFR and ERK was observed between the groups with control and fresh-PRP. Similar results were obtained in the groups with control and FD-PRP (Fig. 3C, D). Conversely, no difference was observed in the levels of phosphorylated PDGFR and ERK between the fresh-PRP and FD-PRP groups. Immunostaining showed a significant proliferation of osteoblasts in both fresh-PRP and FD-PRP (Fig. 4A). Quantification using the MTT assay showed that both fresh-PRP and FDPRP significantly induced the proliferation of osteoblasts, with up to 1.8-times more growth compared with the control group (Fig. 4B). However, no difference was observed

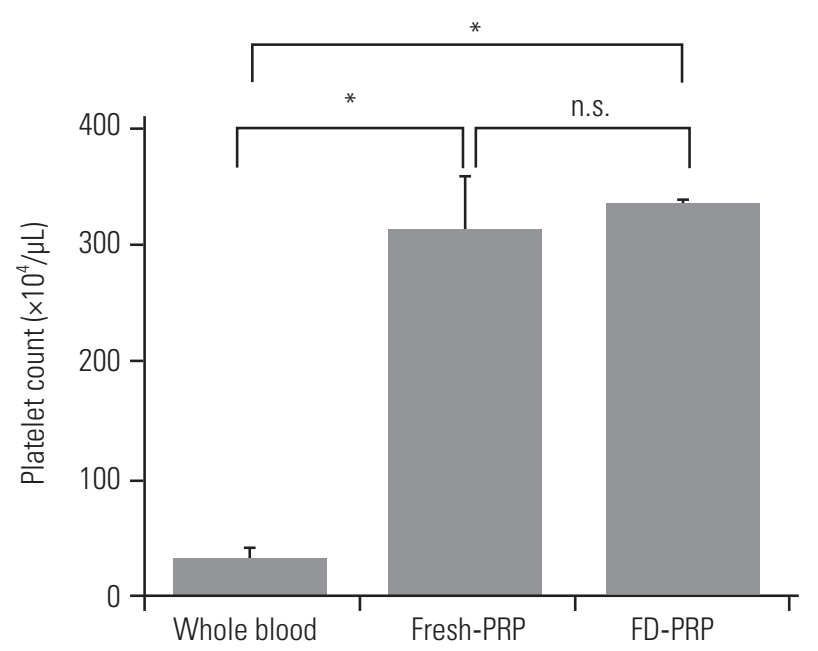

Fig. 2. Platelet counts in PRP. The platelet concentration of fresh-PRP and FD-PRP was 10 -fold higher than that of whole blood. Values are presented mean \pm standard deviation. PRP, platelet-rich plasma; FDPRP, freeze-dried platelet-rich plasma; n.s. not significance. " $p<0.05$ $(n=3)$.
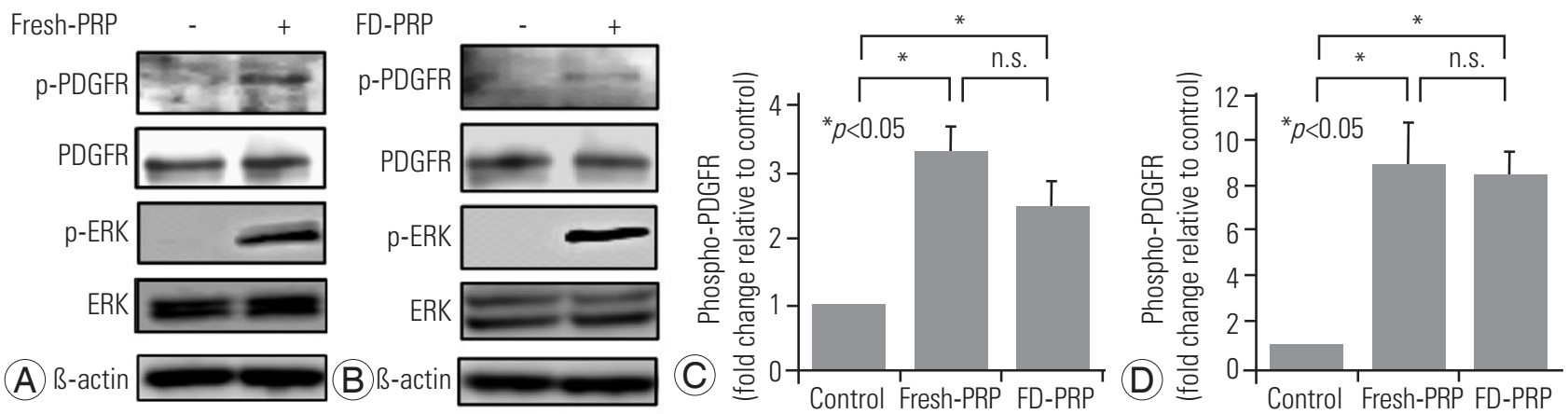

Fig. 3. (A) Western blot analysis indicated that fresh-PRP phosphorylated and activated PDGFR and ERK in the osteoblasts. (B) Western blot showed that FD-PRP phosphorylated PDGFR and ERK in the osteoblasts. (C, D) Fresh-PRP and FD-PRP induced significantly more phosphorylation and activation of PDGFR and ERK compared with the control. However, no difference was observed in the levels of phosphorylated PDGFR and ERK between fresh-PRP and FD-PRP groups. Results are derived from three independent experiments performed in triplicate ( $n=3)$. PRP, platelet-rich plasma; PDGFR, platelet-derived growth factor receptor; ERK, extracellular signal-regulated kinase; FD-PRP, freeze-dried platelet-rich plasma; n.s., not significance. 


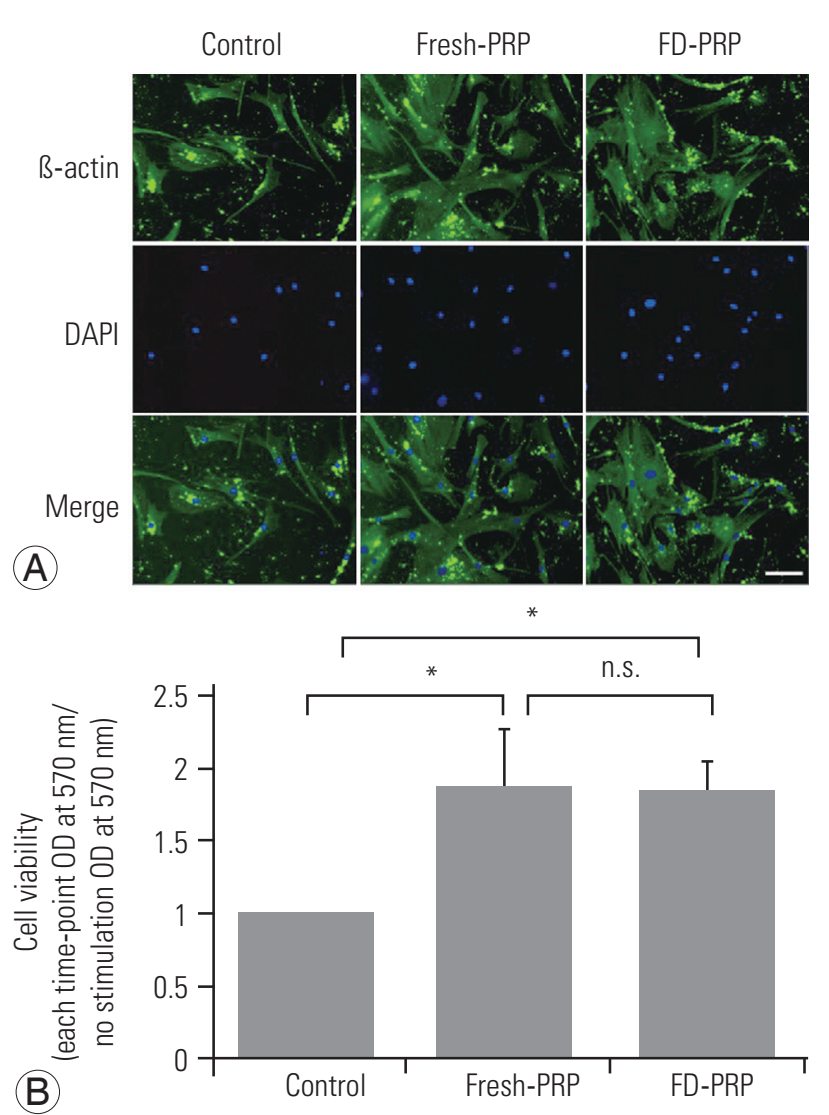

Fig. 4. (A) Immunostaining shows significant proliferation of osteoblasts in both fresh-PRP and FD-PRP. Scale bar $=50 \mu \mathrm{m}$. (B) Quantification of cell viability using MTT assay shows that both fresh-PRP and FD-PRP induce the proliferation of osteoblast approximately 1.8-times more than the control. Values are presented mean \pm standard deviation. PRP, platelet-rich plasma; FD-PRP, freeze-dried platelet-rich plasma; n.s., significance; $0 D$, optical density. ${ }^{*} p<0.05(\mathrm{n}=3)$.

in the proliferation of osteoblasts between the groups with fresh-PRP and FD-PRP.

\section{PDGFR knockdown attenuated the downstream ERK activation by both fresh PRP and FD-PRP}

Next, we evaluated the effects of the knockdown of PDGFR in osteoblasts on ERK activation by PRP. siRNAinduced PDGFR knockdown remarkably suppressed the PDGFR protein expression in western blotting. At each concentration tested, knockdown of osteoblast PDGFR resulted in the attenuation of downstream ERK activation with both fresh PRP (Fig. 5A) and FD-PRP (Fig. 5B).

\section{Discussion}

In this in vitro study, we showed that PDGF in FD-PRP

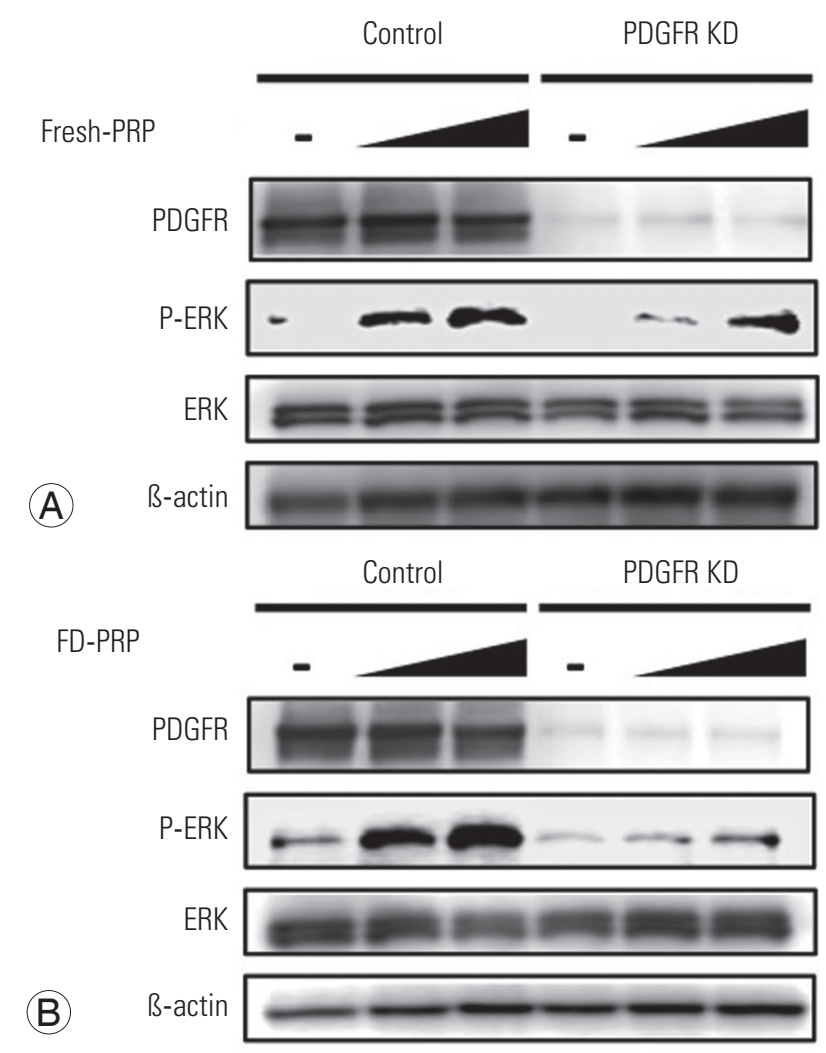

Fig. 5. (A) Fresh-PRP and (B) FD-PRP induce the phosphorylation and activation of ERK in a concentration-dependent manner, which is inhibited in the PDGFR knockdown line. Results are derived from three independent experiments performed in triplicate $(n=3)$. PRP, plateletrich plasma; FD-PRP, freeze-dried platelet-rich plasma; ERK, extracellular signal-regulated kinase; PDGFR, platelet-derived growth factor receptor.

retains its activity and promotes the proliferation of osteoblasts via the PDGFR-mediated ERK signaling pathway in a manner comparable with fresh-PRP even after freezedrying and storing for 4 weeks.

Although we previously reported that FD-PRP promotes bone union in a rat PLF model after 8-week storage, the effectiveness of the GFs included in the FD-PRP was not evaluated. Regarding the validity of the freezedrying procedure, Hanafusa [15] reported that some proteins are inactivated by thawing after freeze-drying and further evaluated the mechanisms for stable or unstable proteins in freeze-dried samples [16]. Nonetheless, in the current study, we showed that PDGF in FD-PRP was almost evenly stored for 4 weeks after freeze-drying. Furthermore, PDGF in FD-PRP induced proliferation of osteoblasts, indicating that it has pharmacological activity even after freeze-drying.

Among the several GFs, we focused on PDGF in the 
current study. In fact, we have already reported PDGF concentration to be more than 50-fold in human PRP in a previous study [9]. Furthermore, PDGF induces osteoblast differentiation by enhancing collagen synthesis and bone cell proliferation as well as tissue repair $[17,18]$. Demirtas et al. [19] reported that isolated PDGF induced two-fold proliferation of osteoblasts in comparison with the control in vitro. In the current study, both fresh-PRP and FD-PRP has similar effects. Both induced approximately two-fold proliferation of osteoblasts. PRP also contains other GFs, such as epidermal growth factor (EGF) and fibroblast GF, which have a similar ability to promote bone union, as does PDGF [20]. Thus, further examination to evaluate whether other GFs in PRP contribute to osteoblast proliferation is required.

As stated above, while PRP is useful because of its concentrated GFs, the effectiveness can vary depending on the platelet concentration. For instance, Yamaguchi et al. [21] evaluated the effects of concentrated PRP on intestinal anastomotic healing in rats. However, the optimal platelet concentration in PRP required for bone fusion is yet under investigation. Graziani et al. [22] investigated the effects of different platelet concentrations in PRP using osteoblasts and concluded that 3-5-fold higher platelet concentration of PRP compared with that of whole blood is effective for tissue repair. In the current study, we obtained up to 10-fold higher platelet concentration of PRP compared with that of whole blood, as shown in Fig. 2. However, since adverse effects, such as exuberant inflammatory reaction [23], may occur at such high platelet concentrations, evaluation of the optimal platelet concentration in PRP should be considered in a future study.

Another issue reported in the studies of PRP is the presence of leukocytes in PRP. Depending on its leukocyte concentration, PRP is classified as leukocyte-poor PRP or leukocyte-rich PRP, and their different activities are highly debated [24]. Yan et al. [25] reported that leukocyte-poor PRP is better at improving tendon healing than leukocyterich PRP and is the preferable option for the clinical treatment of tendinopathy. In the current study, as well as in previous studies, we used leukocyte-poor PRP to avoid the inflammatory action of leukocytes. Although there are few reports of differences in the effects of these PRPs in the bone region, our studies demonstrate that leukocytepoor PRP can promote bone union in vitro and in vivo. Further investigation is required to determine the optimal concentration of leukocytes in PRP.
In the current study, we also investigated the involvement of the MAPKs, which play essential roles in a variety of cell processes by affecting transcriptional or translational regulation, with three known factors, ERK, c-Jun $\mathrm{N}$-terminal kinase (JNK), and p38 [26-28]. ERKs play a central role in cell survival and mitogenic signaling, while the JNK and p38 MAP kinases induce various stress responses, including apoptosis, inflammation, and differentiation [29]. Various studies have reported that ERK acts downstream of the PDGF pathway and regulates cell proliferation [30]. Both fresh-PRP and FDPRP activated PDGFR and its downstream target ERK in osteoblasts, indicating that PDGF concentration in PRP is sufficient to induce the proliferation of osteoblasts via the PDGFR-mediated ERK signaling pathway (Figs. 3, 4). Furthermore, knockdown of osteoblast PDGFR reduced the activation of downstream ERK, showing that PDGF in PRP is required for the proliferation of osteoblasts via the PDGFR-mediated ERK signaling pathway (Fig. 5). These results show that both fresh-PRP and FD-PRP have similar pharmacological activity.

The current study has some limitations. First, we did not evaluate the activity of GFs other than PDGF. Because EGF and other GFs are also reported to promote bone fusion, further investigation of the activity of other GFs is warranted in the future. Second, although we evaluated the effect of PRP on osteoblast proliferation, we did not evaluate the effect of PRP on osteoblast differentiation. Zhao et al. [31] reported that PDGF promotes proliferation and osteogenic differentiation of bone marrow stromal cells via the ERK pathway. Further investigation is required to identify whether FD-PRP also has an osteoblast-differentiating activity. Third, it is also necessary to evaluate the optimal platelet and leukocyte concentration in PRP. Finally, an accurate comparison of the activity between fresh-PRP and FD-PRP is needed.

\section{Conclusions}

In conclusion, we showed that PDGF in FD-PRP is pharmacologically active in vitro after 4 weeks of storage. As concluded in previous reports $[12,13]$, for the clinical application of FD-PRP after extended long-term storage, further investigations are required to determine the effects of FD-PRP on human bone fusion and soft tissue repair. If FD-PRP is shown to be clinically applicable, it could be useful to promote bone fusion after bone fracture surgical 
repair, induce interbody fusion, and provide the appropriate scaffolding for bone formation after spinal surgery without the need of intraoperative blood collection.

\section{Conflict of Interest}

No potential conflict of interest relevant to this article was reported.

\section{Acknowledgments}

This work was supported by JSPS KAKENHI grants (26462228 and 18K09093).

\section{Author Contributions}

HK contributed to original idea, literature review, data collection, statistical analysis, data interpretation, manuscript writing, preparation of figures, revision, and approval of final work. SO, KI, KA, MI, TU, KF, YS, TY, TI, HirK, TA, and TT contributed to original idea, data interpretation, manuscript revision. $\mathrm{SeO}$ contributed to data interpretation and approval of final work. All authors have read and approve the final submitted manuscript.

\section{References}

1. Alsousou J, Thompson M, Hulley P, Noble A, Willett $\mathrm{K}$. The biology of platelet-rich plasma and its application in trauma and orthopaedic surgery: a review of the literature. J Bone Joint Surg Br 2009;91:987-96.

2. Fallouh L, Nakagawa K, Sasho T, et al. Effects of autologous platelet-rich plasma on cell viability and collagen synthesis in injured human anterior cruciate ligament. J Bone Joint Surg Am 2010;92:2909-16.

3. Redler LH, Thompson SA, Hsu SH, Ahmad CS, Levine WN. Platelet-rich plasma therapy: a systematic literature review and evidence for clinical use. Phys Sportsmed 2011;39:42-51.

4. Anitua E, Andia I, Ardanza B, Nurden P, Nurden AT. Autologous platelets as a source of proteins for healing and tissue regeneration. Thromb Haemost 2004;91:415.

5. A Hamid MS, Mohamed Ali MR, Yusof A, George J, Lee LP. Platelet-rich plasma injections for the treatment of hamstring injuries: a randomized controlled trial. Am J Sports Med 2014;42:2410-8.
6. Tietze DC, Geissler K, Borchers J. The effects of platelet-rich plasma in the treatment of large-joint osteoarthritis: a systematic review. Phys Sportsmed 2014;42:27-37.

7. Doria C, Mosele GR, Caggiari G, Puddu L, Ciurlia E. Treatment of early hip osteoarthritis: ultrasound-guided platelet rich plasma versus hyaluronic acid injections in a randomized clinical trial. Joints 2017;5:1525.

8. Kubota G, Kamoda H, Orita S, et al. Platelet-rich plasma enhances bone union in posterolateral lumbar fusion: a prospective randomized controlled trial. Spine J 2019;19:e34-40.

9. Kamoda H, Ohtori S, Ishikawa T, et al. The effect of platelet-rich plasma on posterolateral lumbar fusion in a rat model. J Bone Joint Surg Am 2013;95:110916.

10. Kamoda H, Yamashita M, Ishikawa T, et al. Plateletrich plasma combined with hydroxyapatite for lumbar interbody fusion promoted bone formation and decreased an inflammatory pain neuropeptide in rats. Spine (Phila Pa 1976) 2012;37:1727-33.

11. Vahabi S, Torshabi M, Esmaeil Nejad A. In vitro comparison of the efficacy of TGF- $\beta 1$ and PDGF$\mathrm{BB}$ in combination with freeze-dried bone allografts for induction of osteogenic differentiation in MG63 osteoblast-like cells. J Mater Sci Mater Med 2016;27:182.

12. Shiga Y, Kubota G, Orita S, et al. Freeze-dried human platelet-rich plasma retains activation and growth factor expression after an eight-week preservation period. Asian Spine J 2017;11:329-36.

13. Shiga Y, Orita S, Kubota G, et al. Freeze-dried platelet-rich plasma accelerates bone union with adequate rigidity in posterolateral lumbar fusion surgery model in rats. Sci Rep 2016;6:36715.

14. Aghaloo TL, Moy PK, Freymiller EG. Investigation of platelet-rich plasma in rabbit cranial defects: a pilot study. J Oral Maxillofac Surg 2002;60:1176-81.

15. Hanafusa N. The behavior of hydration water of protein with the protectant in the view of 1HNMR. Dev Biol Stand 1992;74:241-53.

16. Nail SL, Jiang S, Chongprasert S, Knopp SA. Fundamentals of freeze-drying. Pharm Biotechnol 2002;14:281-360.

17. Raines EW, Ross R. Platelet-derived growth factor: I. high yield purification and evidence for multiple 
forms. J Biol Chem 1982;257:5154-60.

18. Shah P, Keppler L, Rutkowski J. A review of platelet derived growth factor playing pivotal role in bone regeneration. J Oral Implantol 2014;40:330-40.

19. Demirtas TT, Goz E, Karakecili A, Gumusderelioglu M. Combined delivery of PDGF-BB and BMP-6 for enhanced osteoblastic differentiation. J Mater Sci Mater Med 2016;27:12.

20. Chandra A, Lan S, Zhu J, Siclari VA, Qin L. Epidermal growth factor receptor (EGFR) signaling promotes proliferation and survival in osteoprogenitors by increasing early growth response 2 (EGR2) expression. J Biol Chem 2013;288:20488-98.

21. Yamaguchi R, Terashima H, Yoneyama S, Tadano S, Ohkohchi N. Effects of platelet-rich plasma on intestinal anastomotic healing in rats: PRP concentration is a key factor. J Surg Res 2012;173:258-66.

22. Graziani F, Cei S, Ducci F, Giuca MR, Donos N, Gabriele $\mathrm{M}$. In vitro effects of different concentration of PRP on primary bone and gingival cell lines. Preliminary results. Minerva Stomatol 2005;54:15-22.

23. Kaux JF, Croisier JL, Leonard P, Le Goff C, Crielaard JM. Exuberant inflammatory reaction as a side effect of platelet-rich plasma injection in treating one case of tendinopathy. Clin J Sport Med 2014;24:150-2.

24. Xu Z, Yin W, Zhang Y, et al. Comparative evaluation of leukocyte- and platelet-rich plasma and pure platelet-rich plasma for cartilage regeneration. Sci Rep 2017;7:43301.
25. Yan R, Gu Y, Ran J, et al. Intratendon delivery of leukocyte-poor platelet-rich plasma improves healing compared with leukocyte-rich platelet-rich plasma in a rabbit achilles tendinopathy model. Am J Sports Med 2017;45:1909-20.

26. Fujisawa T, Takeda K, Ichijo H. ASK family proteins in stress response and disease. Mol Biotechnol 2007;37:13-8.

27. Takeda K, Komuro Y, Hayakawa T, et al. Mitochondrial phosphoglycerate mutase 5 uses alternate catalytic activity as a protein serine/threonine phosphatase to activate ASK1. Proc Natl Acad Sci U S A 2009;106:12301-5.

28. Sekine Y, Takeda K, Ichijo H. The ASK1-MAP kinase signaling in ER stress and neurodegenerative diseases. Curr Mol Med 2006;6:87-97.

29. Li Q, Chen M, Liu H, Yang L, Yang T, He G. The dual role of ERK signaling in the apoptosis of neurons. Front Biosci (Landmark Ed) 2014;19:1411-7.

30. Ying HZ, Chen Q, Zhang WY, et al. PDGF signaling pathway in hepatic fibrosis pathogenesis and therapeutics (Review). Mol Med Rep 2017;16:7879-89.

31. Zhao Y, Zhang S, Zeng D, et al. rhPDGF-BB promotes proliferation and osteogenic differentiation of bone marrow stromal cells from streptozotocininduced diabetic rats through ERK pathway. Biomed Res Int 2014;2014:637415. 